\title{
District hospital surgical capacity in Western Cape Province, South Africa: A cross-sectional survey
}

\author{
P Naidu, MB ChB, MSc; K M Chu, MD, MPH \\ Centre for Global Surgery, Department of Global Health, Faculty of Medicine and Health Sciences, Stellenbosch University, Cape Town, South Africa
}

Corresponding author: KM Chu (kchu@sun.ac.za)

\begin{abstract}
Background. The role of the district hospital (DH) in surgical care has been undervalued. However, decentralised surgical services at DHs have been identified as a key component of universal health coverage. Surgical capacity at DHs in Western Cape (WC) Province, South Africa, has not been described.

Objectives. To describe DH surgical capacity in WC and identify barriers to scaling up surgical capacity at these facilities.

Methods. This was a cross-sectional survey of $33 \mathrm{DHs}$ using the World Health Organization surgical situational analysis tool administered to hospital staff from June to December 2019. The survey addressed the following domains: general services and financing; service delivery and surgical volume; surgical workforce; hospital and operating theatre (OT) infrastructure, equipment and medication; and barriers to scaling up surgical care.

Results. Seven of 33 DHs (21\%) did not have a functional OT. Of the 28 World Bank DH procedures, small WC DHs performed up to $22(79 \%)$ and medium/large DHs up to 26 (93\%). Only medium/large DHs performed all three bellwether procedures. Five DHs (15\%) had a full-time surgeon, anaesthetist or obstetrician (SAO). Of DHs without any SAO specialists, 14 (50\%) had family physicians (FPs). These DHs performed more operative procedures than those without FPs $(p=0.005)$. Lack of finances dedicated for surgical care and lack of surgical providers were the most reported barriers to providing and expanding surgical services.

Conclusions. WC DH surgical capacity varied by hospital size. However, FPs could play an essential role in surgery at DHs with appropriate training, oversight and support from SAO specialists. Strategies to scale up surgical capacity include dedicated financial and human resources.
\end{abstract}

S Afr Med J 2021;111(4):343-349. https://doi.org/10.7196/SAMJ.2021.v111i4.15281

An estimated five billion people lack access to safe surgical care globally, many of whom live in low- and middle-income countries (LMICs).$^{[1]}$ Surgical care, including anaesthesia and obstetrics, could ameliorate or cure up to one-third of the global burden of disease. ${ }^{[2]}$ Improving surgical care in LMICs has become a key component of universal health coverage. Decentralised surgical care has become crucial in advancing surgery on the global health agenda. ${ }^{[3]}$ The district hospital (DH), while often undervalued for its role in surgical care delivery, has been described as the backbone of essential and emergency surgical care (EESC). ${ }^{[2,3]}$ In recent years, there has been increased focus on expanding the role of the $\mathrm{DH}$ to increase geographical access to surgical care for a larger proportion of the population, especially those living in rural areas. However, the DH's role in surgical care has historically been neglected, as evidenced by the lack of investment in resources and healthcare providers to provide surgical, anaesthetic and obstetric (SAO) care at these facilities. ${ }^{[3]}$

South Africa (SA) is considered one of the most unequal countries in the world, with substantial inequities in access to healthcare. ${ }^{[4]}$ While there is a critical shortage of surgeons, the majority work in the private sector, which $80 \%$ of the population are unable to access. ${ }^{[5,6]}$ In SA, SAO care is often provided at secondary- and tertiary-level hospitals because DHs have limited capacity. ${ }^{[7,8]}$ These higher levels of care are usually in urban or semi-urban settings, rendering them less accessible to nearly half the population who live in rural areas. ${ }^{[9]}$ Furthermore, these facilities are often overburdened with long waiting lists for EESC, resulting in delays to care. ${ }^{[10,11]} \mathrm{A}$ recent study ${ }^{[12]}$ reported that one-third of the procedures performed at a tertiary facility could have been performed at a DH. There has been evidence that decentralised surgical services can be cost-effective and improve access to safe and timely EESC..$^{[1,2]}$ Subsequently, in recent years there has been increased focus on health system strengthening, including expanding the role of DHs, which can play a key role in improved community health. A 2020 study ${ }^{[13]}$ demonstrated that $86 \%$ of the population in SA lived within 2 hours of a DH with a surgical provider and a functional operating theatre (OT), but did not measure actual surgical capacity of the facilities.

National and international guidelines on $\mathrm{DH}$-specific procedures exist, but are not widely implemented. The SA National Department of Health $(\mathrm{NDoH}) \mathrm{DH}$ package includes a list of operative procedures, but hospitals are not mandated to report on the volume of these procedures performed ${ }^{[14]}$ The World Bank textbook Disease Control Priorities: Essential Surgery ${ }^{[2]}$ recommends 28 essential DH surgical procedures. In addition, the Lancet Commission on Global Surgery recommended that all DHs be able to provide the bellwether procedures: caesarean section (CS), emergency laparotomy, and irrigation and washout of an open fracture ${ }^{[1,15]}$ Few countries have met these international standards. ${ }^{[7,16,17]}$ In SA, DH surgical capacity has not been systematically measured on a provincial or national scale.

\section{Objectives}

To determine DH surgical capacity and barriers to providing and expanding surgical care in Western Cape (WC) Province, SA.

\section{Methods}

\section{Study design and setting}

This was a cross-sectional survey conducted at all 33 DHs in WC between June and December 2019 (Fig. 1). The World Health Organization (WHO) surgical situational analysis tool, which is freely available ${ }^{[18]}$ was modified for the SA context. 


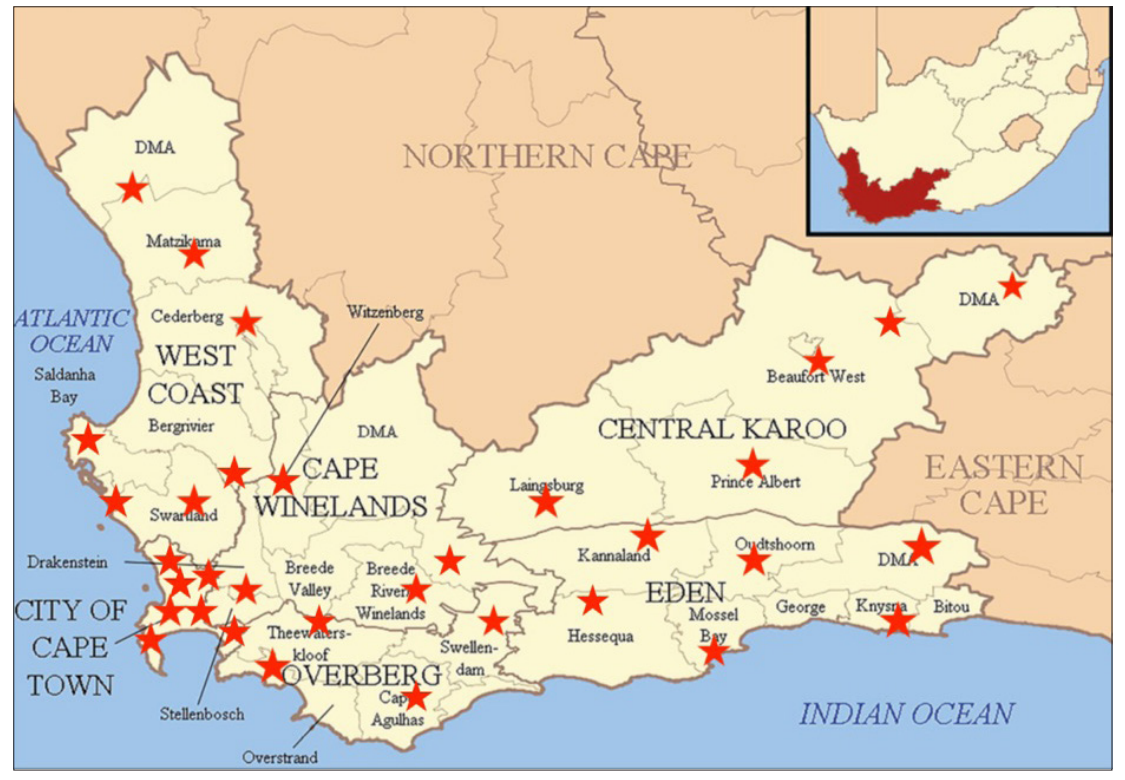

Fig. 1. District hospital locations in Western Cape Province, South Africa.

\section{Participant selection}

All 33 DHs in WC were included in the study. Participants to be interviewed were hospital staff responsible for SAO care, including chief executive officers, medical managers, nursing supervisors, finance officers, doctors and nurses.

\section{Data collection}

The survey was conducted in person by the first author. It covered the following domains: general services and financing; service delivery and surgical volume; surgical workforce; hospital and OT infrastructure, equipment and medication; information systems; and barriers to scaling up surgical care. Data on surgical inpatients, outpatients and operations were extracted from OT registries and hospital databases, and where these data did not exist, best estimates were given by key hospital staff. Questions on barriers to care were responded to by multiple members of SAO teams to minimise selection bias.

\section{Outcome measures}

Facility-level outcome measures included operative capacity, availability of appropriate equipment and medication, monthly surgical volume, procedure type, and number of surgical specialists. Quality outcomes included monthly postoperative mortality rates and postoperative sepsis rates, and perceived barriers to care.

\section{Definitions}

DHs were classified according to SA NDoH guidelines as small ( 0 - 149 beds), medium (150 - 299 beds) and large (300-600 beds). ${ }^{[19]}$ Surgical care included provision of all SAO services in the outpatient department, inpatient department and OT. A non-OT procedure was defined as an operation taking place under local anaesthesia or monitored sedation outside the OT, usually in the emergency department or an outpatient procedure room. Conversely, an OT procedure was defined as an operation taking place in an OT under regional or general anaesthesia. An SAO specialist was defined as a qualified surgeon, anaesthetist or obstetrician. An SAO provider was defined as a doctor who had the skills to provide a component of SAO care but was not a registered SAO specialist. Family physicians (FPs) were included in this category. An obstetric operation was an operative procedure related to obstetric care. A surgical operation was an operative procedure not related to obstetric care (including orthopaedic, paediatric, maxillofacial, trauma and general surgery procedures). Barriers to care were categorised as human resources and training, financial, infrastructural (such as the lack of OTs), and consumable (such as the lack of equipment and medication).

\section{Statistical analysis}

Data were entered in the REDCap (Research Electronic Data Capture) Online Software Tool, version 10.3.5 (Vanderbilt University, USA) and exported to Stata 15 SE (StataCorp, USA) for analysis. Descriptive analyses were conducted. Normally distributed data were determined using a Shapiro-Wilk test. Means were reported and $t$-tests were conducted for normally distributed continuous data.
A one-way analysis of variance was used to analyse group means. Non-normally distributed data were reported as medians with interquartile ranges (IQRs) and Kruskal-Wallis tests were used to compare medians. Chi-square tests were used to compare normally distributed categorical data, and Fischer's exact tests to compare categorical data that were not normally distributed. Results were considered statistically significant at $p<0.05$.

\section{Ethics approval}

Ethics approval was obtained from the University of Cape Town Human Research Ethics Committee (ref. no. 161/2019), the Western Cape Health Impact Committee, and the relevant district health offices.

\section{Results}

\section{Infrastructure}

All 33 DHs in WC were surveyed. Twentyeight DHs were small ( $<150$ beds), 3 were medium (150 - 299 beds), and 2 were large (300 - 600 beds). All DHs reported reliable electricity sources. Thirty (91\%) reported consistent municipal water, and $3(9 \%)$ used boreholes as back-up. Only $1 \mathrm{DH}(3 \%)$ had free staff internet.

\section{Equipment and supplies}

Twelve DHs (36\%) had access to 24-hour X-ray services, $5(15 \%)$ to ultrasound and $1(6 \%)$ to computed tomography scanners; none $(0 \%)$ had magnetic resonance imaging. Eleven (33\%) had on-site laboratory services and the remaining $22(67 \%)$ had nearby access. Twenty-seven (82\%) reported consistent 2-hour access to emergency blood products.

Fifteen DHs (45\%) had designated surgical beds, with a median (IQR) number of beds of 15 (7 - 40). Twenty-six (79\%) had functional OTs, with a median (IQR) of $2(1-2)$. Eighteen $(69 \%)$ had a postanaesthesia recovery unit, while in $8(31 \%)$, patients recovered in the OT. All 26 DHs with functional OTs (100\%) had all the equipment necessary to provide safe surgical and anaesthetic care.

No DH had a designated high-care unit, but 5 (15\%) had high-care beds for increased supervision and monitoring with proximity to ventilators in the emergency department if required. Differences in surgical volume between small and medium/large DHs were statistically significant (Table 1).

\section{Medication supply}

All 26 DHs with functional OTs (100\%) had all the medication necessary to provide safe surgical and anaesthetic care. 


\section{Health information systems}

Thirty-two DHs (97\%) used both electronic and paper-based medical records. Only 1 hospital (3\%) used paper only. Twenty-six (79\%) had a dedicated medical records department, and 22 (67\%) were able to access patients' records across multiple visits. Twentyfive $(83 \%)$ collected postoperative mortality data routinely after every postoperative death. Twenty-eight (85\%) used mobile health platforms, such as Vula or WhatsApp.

\section{Workforce}

Five DHs (15\%) had at least one full-time SAO specialist, and of these, $2(6 \%)$ had at least one of each type of specialist. Twentyfive DHs (76\%) had doctors who provided SAO care (Fig. 2). Of the $28 \mathrm{DHs}$ that did not have any SAO specialists, $14(50 \%)$ had specialist FPs, and the median (IQR) surgical volume in these DHs was $78(60$ - 100) compared with $17(0$ - 55) in DHs without FPs $(p=0.005)$ (Table 2). Three DHs $(9 \%)$ had radiologists and none had pathologists. Twenty-six (79\%) had nurses who worked in the surgical wards, the OT, the post-anaesthesia recovery unit, or as anaesthetic nurses. No DH had non-physician healthcare providers.

\section{Funding}

Twenty DHs (61\%) reported earmarked surgical care budgets. In 17 of the $20(85 \%)$, the surgical budget was $<25 \%$ of the total operational budget.

\section{Procedures}

All DHs performed minor non-OT procedures in the emergency or outpatient departments. These included laceration repair, management of non-displaced fractures, and tube thoracostomy. The most common operative procedures were CS, abscess incision and drainage, and biopsy (Table 3). Seven (21\%) of $33 \mathrm{DHs}$ did not have a functional OT and therefore did not perform operative

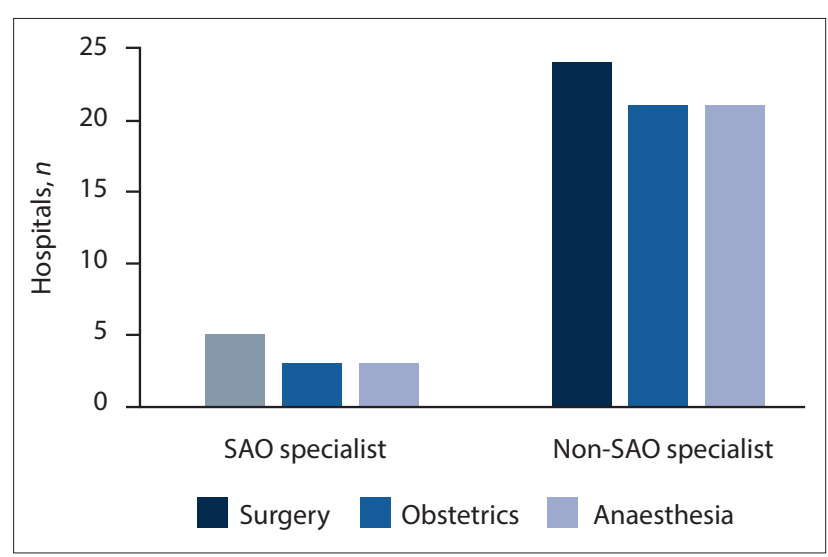

Fig. 2. District hospitals with SAO care specialists and non-SAO specialist providers, Western Cape Province, South Africa. (SAO = surgery, anaesthesia and obstetrics.)

\begin{tabular}{|c|c|c|c|}
\hline & Small $(N=28 ; 85 \%)$ & Medium/large $(N=5 ; 15 \%)$ & $p$-value \\
\hline Catchment population, mean (IQR) & $60484(21588-114950)$ & $650000(391749-900000)$ & $<0.001$ \\
\hline Full-time SAO specialist (at least 1$), n(\%)$ & $1(4)$ & $4(80)$ & $<0.001$ \\
\hline Family physicians, $n$ (\%) & $14(50)$ & $2(40)$ & 0.680 \\
\hline Monthly surgical inpatients (median, IQR) & $33(8-95)$ & $408(365-450)$ & 0.030 \\
\hline Monthly surgical outpatients (median, IQR) & $20(5-73)$ & $360(300-650)$ & 0.044 \\
\hline Monthly surgical volume ( $n$ operative procedures) & & & $<0.001$ \\
\hline Mean & 64 & 355 & \\
\hline None, $n(\%)$ & $7(25)$ & 0 & \\
\hline Low $(0-50), n(\%)$ & $5(18)$ & 0 & \\
\hline Medium (51 - 150), $n(\%)$ & $14(50)$ & 0 & \\
\hline High $(>150), n(\%)$ & $2(7)$ & $5(100)$ & \\
\hline Surgical cases referred to higher-level facility, \% (IQR) & $40(10-75)$ & $15(15-15)$ & 0.062 \\
\hline Emergency operations, \% (IQR) & $20(0-40)$ & $50(35-60)$ & 0.051 \\
\hline
\end{tabular}

Table 2. Surgical volume at district hospitals ${ }^{\star}$ with and without a family physician $(N=28)$ in Western Cape Province, South Africa

\begin{tabular}{|c|c|c|c|}
\hline & $\begin{array}{l}\text { Without family } \\
\text { physician }(N=14)\end{array}$ & $\begin{array}{l}\text { With family physician } \\
(N=14)\end{array}$ & $p$-value \\
\hline Facility by hospital size, $n$ (\%) & & & 0.309 \\
\hline Small & $14(100)$ & $13(93)$ & \\
\hline Medium & 0 & $1(7)$ & \\
\hline Large & 0 & 0 & \\
\hline \multicolumn{4}{|c|}{ Facility by monthly surgical volume ( $n$ operative procedures) } \\
\hline None, $n(\%)$ & $6(42)$ & $1(7)$ & \\
\hline Low $(0-50), n(\%)$ & $4(29)$ & $1(7)$ & \\
\hline Medium (51 - 150), $n(\%)$ & $4(29)$ & $9(64)$ & \\
\hline High $(>150), n(\%)$ & 0 & $3(22)$ & \\
\hline Mean (IQR) & $17(0-55)$ & $78(60-100)$ & 0.005 \\
\hline
\end{tabular}




\begin{tabular}{|c|c|c|}
\hline Procedure & $\begin{array}{l}\text { Small hospital (number of } \\
\text { procedures), median (IQR) }\end{array}$ & $\begin{array}{l}\text { Medium/large hospital (number } \\
\text { of procedures), median (IQR) }\end{array}$ \\
\hline \multicolumn{3}{|l|}{ Non-operating room procedures } \\
\hline Laceration repair* & $88(48-233)$ & $350(300-670)$ \\
\hline Management of non-displaced fractures ${ }^{*}$ & $30(10-48)$ & $50(40-50)$ \\
\hline Tube thoracostomy ${ }^{\dagger}$ & $10(4-18)$ & $10(5-34)$ \\
\hline \multicolumn{3}{|l|}{ Obstetric procedures } \\
\hline Caesarean section ${ }^{\dagger \ddagger}$ & $10(0-25)$ & $100(53-146)$ \\
\hline Surgical termination of pregnancy ${ }^{\dagger}$ & $4(0-9)$ & $20(14-25)$ \\
\hline Colposcopy $^{\dagger}$ & $0(0-9)$ & $18(10-50)$ \\
\hline Tubal ligation ${ }^{\dagger}$ & $3(0-6)$ & $15(5-15)$ \\
\hline Vacuum extraction or forceps delivery ${ }^{\dagger}$ & $1(0-2)$ & $5(0-26)$ \\
\hline Ectopic pregnancy ${ }^{\dagger}$ & $1(0-3)$ & $11(9-15)$ \\
\hline Vasectomy $^{\dagger}$ & $0(0-2)$ & $4(0-4)$ \\
\hline Hysterectomy ${ }^{\dagger}$ & $0(0-0)$ & $5(4-6)$ \\
\hline \multicolumn{3}{|l|}{ Surgical procedures } \\
\hline Abscess incision and drainage ${ }^{*}$ & $10(7-16)$ & $25(20-30)$ \\
\hline Biopsy & $9(3-15)$ & $12(8-15)$ \\
\hline Wound debridement & $5(1-8)$ & $12(10-23)$ \\
\hline Removal of foreign body & $3(1-8)$ & $2(2-2)$ \\
\hline Male medical circumcision & $1(0-4)$ & $2(2-3)$ \\
\hline Suprapubic catheterisation ${ }^{\dagger}$ & $1(0-3)$ & $1(0-3)$ \\
\hline Repair of intestinal perforation ${ }^{\dagger}$ & $0(0-0)$ & $2(1-4)$ \\
\hline Appendicectomy ${ }^{\dagger}$ & $0(0-0)$ & $11(10-12)$ \\
\hline Release of bowel obstruction ${ }^{\dagger}$ & $0(0-0)$ & $1(1-2)$ \\
\hline Colostomy/ileostomy $^{\dagger}$ & $0(0-0)$ & $2(3-4)$ \\
\hline Cholecystectomy $^{\dagger}$ & $0(0-0)$ & $12(9-17)$ \\
\hline Hernia repair ${ }^{\dagger}$ & $0(0-3)$ & $14(11-16)$ \\
\hline Hydrocelectomy $^{\dagger}$ & $0(0-1)$ & $3(1-4)$ \\
\hline Drainage of septic arthritis ${ }^{\dagger}$ & $0(0-0)$ & $2(1-3)$ \\
\hline Debridement of osteomyelitis ${ }^{\dagger}$ & $0(0-0)$ & $0(0-1)$ \\
\hline \multicolumn{3}{|l|}{ Trauma procedures } \\
\hline Fracture reduction ${ }^{\dagger}$ & $10(5-15)$ & $50(17-50)$ \\
\hline Irrigation and debridement of open fractures ${ }^{\dagger \ddagger}$ & $1(0-5)$ & $25(16-38)$ \\
\hline Surgical airway ${ }^{\dagger}$ & $0(0-0)$ & $0(0-0)$ \\
\hline Trauma laparotomy ${ }^{\ddagger}$ & $0(0-0)$ & $4(0-5)$ \\
\hline Placement of an external fixator ${ }^{\dagger}$ & $0(0-0)$ & $4(1-10)$ \\
\hline Escharotomy/fasciotomy ${ }^{\dagger}$ & $0(0-0)$ & $0(0-2)$ \\
\hline Limb amputations $^{\dagger}$ & $0(0-2)$ & $16(10-16)$ \\
\hline Skin graft ${ }^{\dagger}$ & $0(0-1)$ & $5(3-5)$ \\
\hline Burr holes $^{\dagger}$ & $0(0-0)$ & $0(0-0)$ \\
\hline $\begin{array}{l}\text { IQR = interquartile range; DH = district hospital. } \\
\text { *World Bank primary health clinic level procedure. } \\
\text { 'World Bank DH level procedure. } \\
\text { tLancet Commission on Global Surgery bellwether procedure. }\end{array}$ & & \\
\hline
\end{tabular}

procedures (zero monthly procedures); all these DHs were classified as small. Small DHs with a functional OT performed a mean of 64 procedures a month compared with 335 by medium/large facilities $(p<0.001)$. Small DHs performed $22(79 \%)$ and medium/large DHs 26 (96\%) of the 28 World Bank DH procedures. Of the three bellwether procedures, small DHs consistently performed only CS, compared with medium/large DHs, which performed irrigation and washout of open fractures and trauma laparotomies in addition to CS.

\section{Barriers to care}

Barriers to surgical care include both human (Fig. 3) and non-human (Fig. 4) resource barriers to providing and expanding surgical care. Lack of nursing staff for scrub care, post-anaesthesia recovery and surgical ward care were the most cited human resource barriers, with $27 \mathrm{DHs}(82 \%)$ reporting a lack of nursing staff essential for the delivery of surgical care. Twenty-four DHs (73\%) reported a lack of surgical and anaesthesia providers and $19(58 \%)$ reported a lack of obstetrics providers (Fig. 3). A lack of financial resources $(n=28 ; 85 \%)$ was the most common non-human resource barrier (Fig. 4). Other systemic barriers included perceived out-of-pocket expenditure incurred by patients to access surgical care $(n=16 ; 48 \%)$ and a lack of surgical equipment ( $n=16 ; 48 \%)$, OTs $(n=13 ; 39 \%)$, anaesthetic equipment $(n=9 ; 27 \%)$ and postoperative medicines $(n=7 ; 15 \%)$ (Fig. 4$)$.

\section{Discussion}

DHs can play a central role in universal health coverage, including 


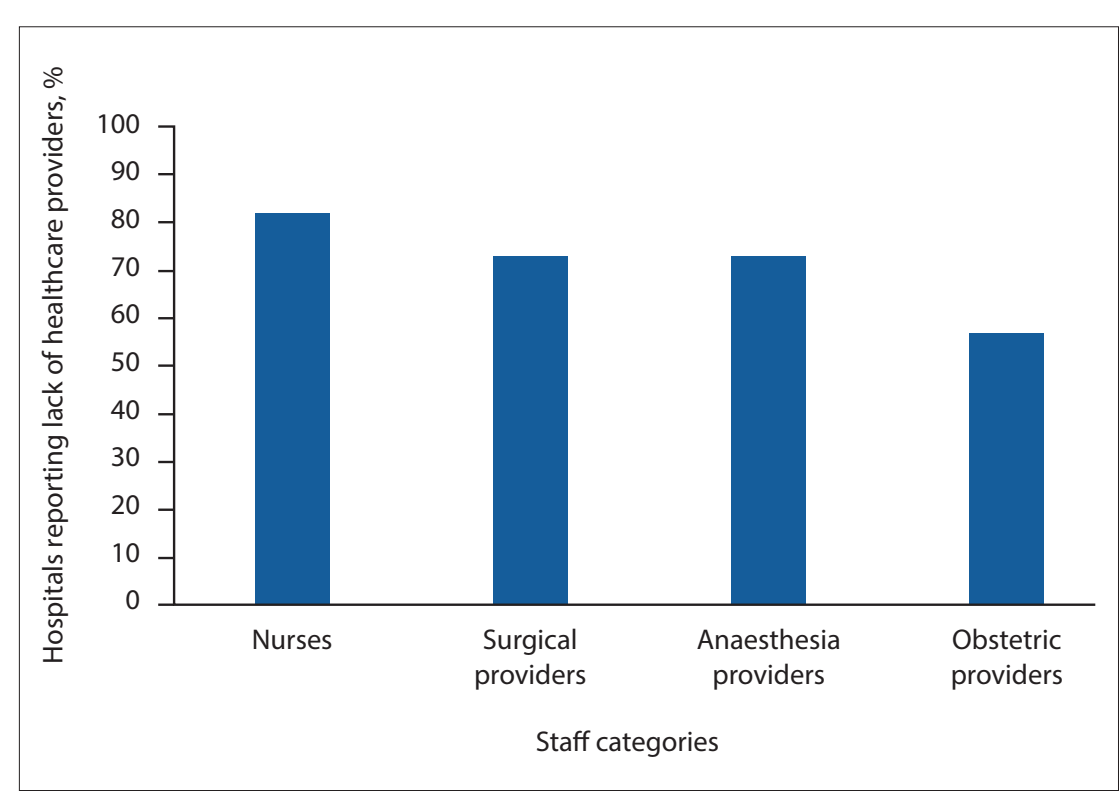

Fig. 3. Human resource barriers to providing and expanding surgical care at district hospitals in Western Cape Province, South Africa.

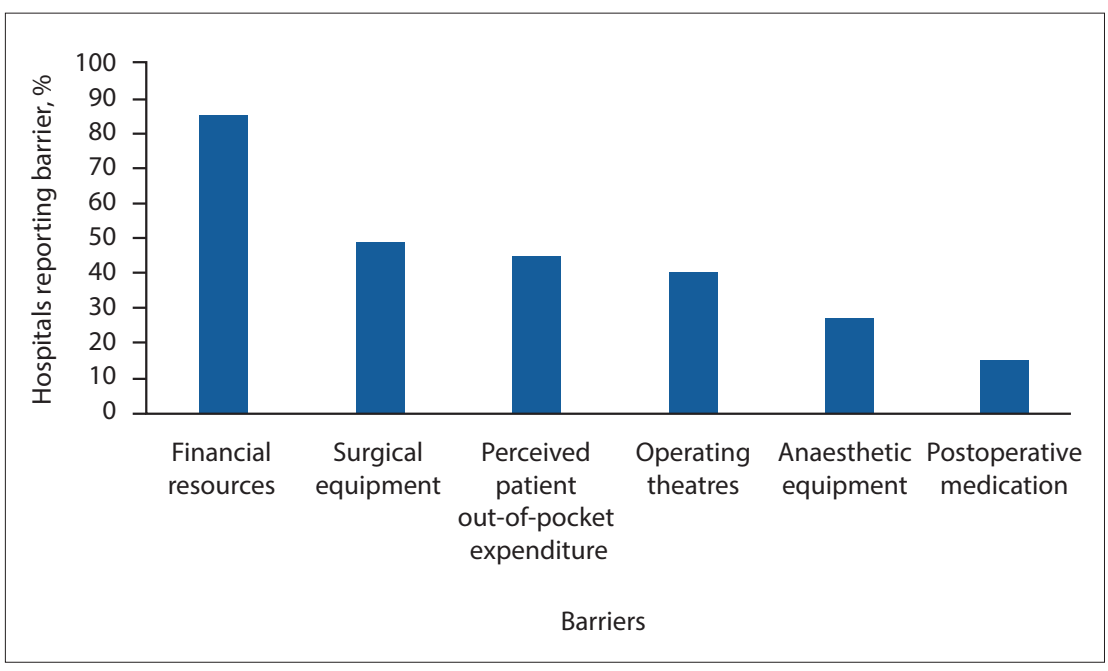

Fig. 4. Non-human resource barriers to providing and expanding surgical care at district hospitals in Western Cape Province, South Africa.

provision of decentralised surgical services. ${ }^{[3]}$ There are many reasons to strengthen surgical care at DHs: providing care closer to patients' homes, reducing patient outof-pocket expenditure to reach care, and decreasing the burden on higher-level facilities and waiting lists, ultimately resulting in more timely surgical care. ${ }^{[3]}$ For a $\mathrm{DH}$ to deliver quality surgical care, it must have relevant infrastructure, equipment and medication, SAO providers, and an earmarked budget for surgical services. Our study found that the majority of WC DHs had good supportive infrastructure such as reliable electricity, running water, laboratory services and emergency blood products. However, $\sim 20 \%$ did not have functional OTs, and of the hospitals that did, $31 \%$ did not have dedicated post-anaesthesia units.
The lack of post-anaesthesia units in onethird of facilities performing surgical care is of great concern, considering findings from the African Surgical Outcomes Study that patients in Africa are twice as likely to die postoperatively compared with the global average. ${ }^{[20]}$ Surveillance in the postoperative period is imperative and requires adequately trained nursing staff and dedicated postanaesthesia units.

DHs, even small ones, should provide some EESC capacity. Defining a minimum procedural basket for $\mathrm{DHs}$ is necessary and should be an objective of the recently established South African National Surgical Obstetric Anaesthesia Plan Task Team. This basket of care can and should be modified for different DHs based on staffing and distance to higher-level hospitals. Large metropolitan
DHs may have nearby referral facilities with fully qualified surgeons who are available for outreach or to assist in the OT and may therefore be able to provide more complex baskets of care. On the other hand, small DHs, especially ones in rural areas, may need to stick to the minimum basket and establish strong referral pathways to higher-level hospitals for all other surgical conditions.

The most common operative procedures were CS, abscess incision and drainage, and biopsy. The majority of WC DHs are small and performed 22 out of 28 (79\%) of the World Bank DH procedures. Of the three bellwether procedures, small DHs only performed one, CS, consistently. Small DHs performed mostly elective operations, despite a recommendation from the World Health Assembly that decentralised surgical services should provide emergency care. ${ }^{[21]}$ While Western Cape DH surgical capacity falls short of the World Bank international guidelines, the surgical capacity is similar to other LMICs, such as Mongolia, where the most common $\mathrm{DH}$ procedures are abscess incision and drainage, suturing, and wound debridement. ${ }^{[22]}$ However, these procedures are often performed in the emergency department in SA DHs. In Sierra Leone DHs, the most common procedures were CS, appendicectomies, and inguinal hernia repairs - procedures that are mostly performed at medium/large DHs in SA. ${ }^{[23]}$

Few WC DHs had SAO specialists; however, those that had at least one FP performed twice as many operative procedures as those that did not have FPs. FPs are trained in a variety of surgical competencies, and most FP training programmes incorporate 18 of the 28 World Bank first-level procedures. With a national target to staff every $\mathrm{DH}$ facility with an FP, this cadre could potentially play a vital role in training, supporting and improving EESC at the DH level. ${ }^{[24]}$ Surgical procedures may be challenging to provide at small DHs because of the limited number of trained surgical providers. FPs can play an important role; however, if a small DH only has one, this may not be sufficient to deliver safe surgical care, which requires at the minimum one surgical and one anaesthetic provider. In addition, rotating teams are needed to sustain these efforts. A previous study reported that in small rural DHs, a single provider often takes on the role of both surgeon and anaesthetist in one-third of emergency CSs, which predisposes to complications and does not make for a safe surgical environment. ${ }^{[25]}$

There are numerous barriers to performing surgery at DHs. Studies in other 
sub-Saharan African settings have shown similar barriers to DH surgical care, such as a lack of infrastructure and appropriately trained SAO providers. ${ }^{[2,27]}$ In our study, a lack of skilled nursing staff was cited as the most common human resource barrier to providing and expanding surgical services. Without nurses in the OT, in the postoperative recovery unit and on surgical wards, safe surgical care is not possible.

Barriers to scaling up surgical services included a lack of appropriately trained doctors with SAO skills and lack of finances for surgical care and providers. DHs need to identify the minimum number of healthcare providers required to create core surgical teams and identify accompanying training and supervision. However, trained staff to provide SAO care cannot be increased in isolation. Staff require the appropriate equipment and medications to deliver safe SAO care, as noted in a Zambian study showing that an increase in workforce but a decrease in equipment and consumables for SAO care did not result in an increase in surgical volume. ${ }^{[16]}$ Associate physicians, or mid-level providers, are critical in the provision of SAO care in many sub-Saharan African countries. ${ }^{[28,29]}$ While associate physicians (clinical associates) have been trained for the provision of and identified as a key component of surgical and anaesthesia care in $\mathrm{SA},{ }^{[30]}$ our study did not report any associate physicians involved in surgical care at the DHs in WC.

The DH was recently deemed the 'neglected hospital' in the health system in LMICs. ${ }^{[3]}$ Universal health coverage and prioritisation of surgery on the global health agenda will only be possible if greater investments are made in DHs ${ }^{[3]}$ Increasing providers and equipment for safe SAO care requires funding, which has historically not been prioritised for surgical care in SA and other LMICs. ${ }^{[28]}$ Only 25\% of DHs in our study had earmarked budgets for surgical services, and this was identified as the greatest non-human resource barrier to providing and expanding surgical capacity. Funding for national surgical plans is essential to scaling up quality SAO care and ensuring that increased access to $\mathrm{SAO}$ care is prioritised, particularly at the $\mathrm{DH}$ level. ${ }^{[3,29]}$ With the impending National Health Insurance plan in SA, understanding surgical capacity and addressing barriers to accessing surgical care are crucial.

\section{Study strengths and limitations}

The accuracy of surgical volume was limited by the lack of quantitative data for non-OT and OT procedures from some facilities. To limit recall bias, best estimates from more than one healthcare provider were used if procedural logbooks were not available. Barriers to scaling up surgical capacity were respondent perceptions and not objectively measured. Additionally, patient out-of-pocket expenditure was reported from the healthcare provider perspective and not routinely measured in this study. Despite these limitations, this study was the first to systematically describe the surgical capacity of all DHs in WC using a WHO-validated surgical situational analysis tool.

\section{Recommendations}

A basic DH procedural list needs to be established that is locally relevant and adapted by local stakeholders. Once the scope of surgical output is clearly defined, further studies can be undertaken to identify and mitigate barriers to scaling up and improving the quality of decentralised surgical services. At a recent workshop on surgical care in rural hospitals, DH doctors reported that challenges to providing or scaling up surgical capacity at DHs included poor communication with and lack of support from SAO specialists working at referral hospitals. ${ }^{[31]} \mathrm{DH}$ doctors felt they would be criticised if they attempted surgical procedures, even if they had relevant training and experience. Formalised mentorship from specialists, outreach and in-reach opportunities, and clear lines of communication for referrals were identified as solutions to improve rural DH surgical capacity. ${ }^{[30]}$ Training courses for non-SAO specialists have also been recommended in other African studies to address the shortage of specialists and facilitate improved relationships between the DHs and higher levels of care, especially in rural areas. ${ }^{[25]}$ Increased funding and financial support for surgical and anaesthesia care, increased access to training, and support from higher levels of care are all crucial to increase surgical capacity at DHs.

\section{Conclusions}

Surgical capacity at WC DHs was highly variable, with the lowest capacity at small facilities. All DHs, regardless of size, should be able to provide a minimum procedural basket of surgical care. The composition of this basket should be contextually relevant and take into account surgical needs and population size. The presence of FPs was associated with increased surgical output, and this specialist cadre could contribute to the scale-up of DH surgical capacity. Strengthening DH services, including the provision of EESC, is essential to universal health coverage in SA, and strategies to improve care at this level are needed.

\section{Declaration. None.}

Acknowledgements. The authors would like to acknowledge the staff at the $33 \mathrm{DH}$ for their contributions to the study.

Author contributions. PM and KMC contributed equally to the study design, data collection, data analysis, and write-up and critical review of the manuscript.

Funding. This research was funded by the National Research Foundation Competitive Support for Unrated Researchers (UID 120189).

Conflicts of interest. None.

1. Meara JG, Leather AJ, Hagander L, et al. Global Surgery 2030: Evidence and solutions for achieving health, welfare and economic development Lancet 2015;386(9993).569-624. https//doi org/10.1016/j.

surg. 2015.02 .009

World Bank. Disease conto
(accessed 25 August 2020). Rajbhandari R, McMahon DE, Rhatigan JJ, Farmer PE. The neglected hospital - the district hospital's central role in global health care delivery. N Engl J Med 2020;382(5):397-400. https://doi.org/10.1056/ nejmp 1911298

4. Harris B, Goudge J, Ataguba JE, et al. Inequities in access to health care in South Africa.J Public Health Policy 2011;32(1):102-123. https://doi.org/10.1057/jphp.2011.35

5. Dell AJ, Kahn D. Where are general surgeons located in South Africa? S Afr J Surg 2018;56(1):12-18. https://doi.org/10.17159/2078-5151/2018/v56n1a2393

6. Kahn D, Pillay S, Veller M, et al. General surgery in crisis - the critical shortage. S Afr J Surg 2006;44(3):88-94.

Tefera A, Lutge E, Sartorius B, et al. The operative output of district hospitals in KwaZulu-Natal Province is heavily skewed toward obstetrical care. World J Surg 2019;43(7):1653-1660. https://doi. org/10.1007/s00268-019-04985-1

8. Voss M, Duvenage R. Operative surgery at the district hospital. S Afr Med J 2011;101(8):521-522.

9. Kok P, Collinson M. Migration and urbanisation in South Africa. Pretoria: Statistics South Africa, 2006. http://www.statssa.gov.za/publications/Report-03-04-02/Report-03-04-02.pdf (accessed 29 August 2006.).

10. South African Government. Gauteng Health on waiting list for operations. 31 March 2015. https:// www.gov.za/speeches/gauteng-health-waiting-list-operations-31-mar-2015-0000 (accessed 14 August

11. Bhuiyan M, Mavhungu R, Machowski A. Provision of an emergency theatre in tertiary hospitals is cost-effective: Audit and cost of cancelled planned elective general surgical operations at Pietersburg Hospital, Limpopo Province, South Africa. S Afr Med J 2017;107(3):239-242. https://doi.org/10.7196/ SAMJ.2017.v107i3.10687

12. Van Straten S, Stannard C, Bulabula J, et al. A third of patients treated at a tertiary-level surgical service could be treated at a secondary-level facility. S Afr Med J 2017;107(9):788-790. https://doi.org/10.7196/ SAMJ.2017.v107i9.12090

13. Chu KM, Dell AJ, Moultrie $\mathrm{H}$, et al. A geospatial analysis of two-hour surgical access to district hospitals in South Africa. BMC Health Serv Res 2020;20(1):744. https://doi.org/10.1186/s12913-020-05637-0

14. Department of Health, South Africa. A district hospital service package for South Africa: A set of norms and standards. March 2002. http://www.kznhealth.gov.za/norms.pdf (accessed 3 March 2020). 5. O'Neill KM, Greenberg SL, Cherian M, et al. Bellwether procedures for monitoring and planning essential surgical care in low- and middle-income countries: Caesarean delivery, laparotomy, and essential surgical care in low- and middle-income countries: Caesarean delivery, laparotomy, and
treatment of open fractures. World J Surg 2016;40(11):2611-2619. https://doi.org/10.1007/s00268$016-3614-y$ 
16. Cheelo M, Brugha R, Bijlmakers L, Kachimba J, McCauley T, Gajewski J. Surgical capacity at district hospitals in Zambia: From 2012 to 2016. World J Surg 2018;42(11):3508-3513. https://doi.org/10.1007/ s00268-018-4678-7

17. Muhirwa E, Habiyakare C, Hedt-Gauthier BL, et al. Non-obstetric surgical care at three rural district hospitals in Rwanda: More human capacity and surgical equipment may increase operative care. World hospitals in Rwanda: More human capacity and surgical equipment may ince
J Surg 2016;40(9):2109-2116. https://doi.org/10.1007/s00268-016-3515-0

18. Surg 2016;40(9):2109-2116. https://doi.org/10.1007/s00268-016-3515-0
World Health Organization. WHO Integrated Management for Emergency \& Essential Surgical Care (IMEESC) toolkit. 2015. https://www.who.int/surgery/publications/who_eesc_situationanalysistool. pdf (accessed 21 August 2020)

19. National Department of Health, South Africa. National Health Act, 2003: Policy on the Management of Public Hospitals. 12 August 2011. https://static.pmg.org.za/docs/110812hospital-mmanagement. pdf (accessed 3 August 2020).

20. Biccard BM, Madiba TE, Kluyts H-L, et al. Perioperative patient outcomes in the African Surgical Outcomes Study: A 7-day prospective observational cohort study. Lancet 2018;391(10130):1589-1598. https://doi.org/10.1016/S0140-6736(18)30001-1

21. Price R, Makasa E, Hollands M. World Health Assembly Resolution WHA68.15: 'Strengthening emergency and essential surgical care and anesthesia as a component of universal health coverage' - addressing the public health gaps arising from lack of safe, affordable and accessible surgical and - addressing the public health gaps arising from lack of sa

22. Osen H, Chang D, Choo S, et al. Validation of the World Health Organization tool for situational analysis to assess emergency and essential surgical care at district hospitals in Ghana. World J Surg 2011;35(3):500-504. https://doi.org/10.1007/s00268-010-0918-

23. Kingham TP, Kamara TB, Cherian MN, et al. Quantifying surgical capacity in Sierra Leone: A guide for improving surgical care. Arch Surg 2009;144(2):122-127. https://doi.org/10.1001/archsurg.2008.540

24. Chu K, Naidu P, Reid S, et al. The role of family physicians in emergency and essential surgical care in the district health system in South Africa. S Afr Fam Pract 2020;62(1):e1-e3. https://doi.org/10.4102/ safp.v62i1.5117
25. Reid S, Chabikuli N, Jaques P, Fehrsen G. The procedural skills of rural hospital doctors. S Afr Med J 1999;89(7):769-774

26. Kalu QN, Eshiet AI, Ukpabio EI, Etiuma AU, Monjok E. A rapid need assessment survey of anaesthesia and surgical services in district public hospitals in Cross River State, Nigeria. Br J Med Pract 2014:7(4):a733.

27. Vansell HJ, Schlesinger JJ, Harvey A, Rohde JP, Persaud S, McQueen KA. Anaesthesia, surgery, obstetrics, and emergency care in Guyana. J Epidemiol Glob Health 2015;5(1):75-83. https://doi org/10.1016/j.jegh.2014.08.003

28. Henry JA, Frenkel E, Borgstein E, Mkandawire N, Goddia C. Surgical and anaesthetic capacity of hospitals in Malawi: Key insights. Health Policy Plan 2015;30(8):985-994. https://doi.org/10.1093/ heapol/czul02

29. Reddy CL, Peters AW, Jumbam DT, et al. Innovative financing to fund surgical systems and expan surgical care in low-income and middle-income countries. BMJ Glob Health 2020;5(6):e002375. https://doi.org/10.1136/bmjgh-2020-002375

30. Chu KM, Naidu P, Hendriks HJ, et al. Surgical care at rural district hospitals in low- and middleincome countries: An essential component of universal health coverage. Rural Remote Health 2020;20(2):1. https://doi.org/10.22605/rrh5920

31. Fraizyngier V, Odingo G, Barone M, Perchal P, Pavin M. Safety of adult medical male circumcision performed by non-physician clinicians in Kenya: A prospective cohort study. Glob Health Sci Pract performed by non-physician clinicians in Kenya: A prospectiv

Accepted 27 October 2020. 